\title{
High Sensitivity Plasmonic Sensor Based on Fano Resonance with Inverted U-Shaped Resonator
}

\author{
Gongli Xiao ${ }^{1}$, Yanping $\mathrm{Xu}^{1}{ }^{1}$, Hongyan Yang ${ }^{2,3, * \mathbb{C}}$, Zetao Ou ${ }^{1}$, Jianyun Chen ${ }^{1}$, Haiou Li ${ }^{1}$, Xingpeng Liu ${ }^{1}$, \\ Lizhen Zeng ${ }^{4}$ and Jianqing $\mathrm{Li}^{5}{ }^{5}$ \\ 1 Guangxi Key Laboratory of Precision Navigation Technology and Application, \\ Guilin University of Electronic Technology, Guilin 541004, China; xiaogl.hy@guet.edu.cn (G.X.); \\ 19022304015@mails.guet.edu.cn (Y.X.); 19022202023@mails.guet.edu.cn (Z.O.); \\ 19022202002@mails.guet.edu.cn (J.C.); lihaiou@guet.edu.cn (H.L.); tadyliu@guet.edu.cn (X.L.) \\ 2 Guangxi Key Laboratory of Automatic Detecting Technology and Instruments, \\ Guilin University of Electronic Technology, Guilin 541004, China \\ 3 School of Electronic Engineering and Automation, Guilin University of Electronic Technology, \\ Guilin 541004, China \\ 4 Graduate School, Guilin University of Electronic Technology, Guilin 541004, China; zlzh@guet.edu.cn \\ 5 Guangdong-Hong Kong-Macao Joint Laboratory for Intelligent Micro-Nano Optoelectronic Technology, \\ Foshan University, Foshan 528225, China; jqli@must.edu.mo \\ * Correspondence: hyyang@guet.edu.cn; Tel.: +86-137-0783-2801
}

Citation: Xiao, G.; Xu, Y.; Yang, H.; Ou, Z.; Chen, J.; Li, H.; Liu, X.; Zeng, L.; Li, J. High Sensitivity Plasmonic Sensor Based on Fano Resonance with Inverted U-Shaped Resonator. Sensors 2021, 21, 1164. https://doi.org/10.3390/s21041164

Academic Editor: Shivananju Bannur Nanjunda

Received: 11 January 2021

Accepted: 4 February 2021

Published: 7 February 2021

Publisher's Note: MDPI stays neutral with regard to jurisdictional claims in published maps and institutional affiliations.

Copyright: (c) 2021 by the authors. Licensee MDPI, Basel, Switzerland. This article is an open access article distributed under the terms and conditions of the Creative Commons Attribution (CC BY) license (https:// creativecommons.org/licenses/by/ $4.0 /)$.

\begin{abstract}
Herein, we propose a tunable plasmonic sensor with Fano resonators in an inverted Ushaped resonator. By manipulating the sharp asymmetric Fano resonance peaks, a high-sensitivity refractive index sensor can be realized. Using the multimode interference coupled-mode theory and the finite element method, we numerically simulate the influences of geometrical parameters on the plasmonic sensor. Optimizing the structure parameters, we can achieve a high plasmonic sensor with the maximum sensitivity for $840 \mathrm{~nm} /$ RIUand figure of merit for $3.9 \times 10^{5}$. The research results provide a reliable theoretical basis for designing high sensitivity to the next generation plasmonic nanosensor.
\end{abstract}

Keywords: Fano resonance; metal-dielectric-metal; finite element method; plasmonic nanosensor

\section{Introduction}

Surface plasmonic sensing technology is considered one of the most promising plasmonic applications, which have important chemical analysis applications, biological monitoring, and other fields [1-3]. As a branch of surface plasmonic research, surface plasmon Fano resonance has become a hot spot in sensing due to its steep asymmetric response spectrum, field enhancement effect, high refractive index sensitivity, and a high figure of merit $[4,5]$. This Fano resonance is caused by the coupling of the narrowband dark mold and the bright broadband mold in the structure. Unlike the traditional Lorentz line, Fano resonance has sharp asymmetry, which is very sensitive to the surrounding environment's variation and structural parameters. It can obtain higher sensitivity and a figure of merit, thus in the refractive index, sensing aspect is deeply concerned [6,7]. In 2010, Boris et al. [8] reviewed the Fano resonance in plasmonic nanostructures, metal photonic crystals, and metamaterials, indicating that Fano resonance can be applied to label-free detection [9], filter $[10,11]$, sensor $[12,13]$, slow light device $[14,15]$, optical switch [16], etc. Studies have shown that surface plasmon polaritons (SPP) are the electromagnetic wave produced by the interaction between free electrons and lightwave on the metal surface. The electromagnetic wave propagates along with the interface between the metal and the dielectric and attenuates exponentially in the direction perpendicular to the metal dielectrics interface. [17,18]. Using the unique light properties of surface plasmon breaks the diffraction limit of traditional optics. Optical devices have low loss, small size and long propagation distance, 
easy integration, and other advantages $[19,20]$. In recent years, researchers have used the Fano resonance generated by metal nanostructures to conduct a lot of research on their nanostructures' propagation characteristics and sensing properties. For example, in 2016, Zhang et al. [21] reported a refractive index sensor based on metal-insulator-metal (MIM) waveguide coupled double rectangular cavities. The Fano resonance peak depended on the mode coupling between the double rectangular cavities.

The results show that the maximum sensitivity (S) of $596 \mathrm{~nm} / \mathrm{RIU}$, the maximum FOM of less than 10. In 2017, Zhao et al. [22] proposed that the tunable Fano resonance can be realized by coupling an asymmetric structure of a ring resonator on the side of the metal baffle. Changing the ring resonator's inner radius, the maximum sensitivity, and the ultimate figure of merit can be up to $718 \mathrm{~nm} / \mathrm{RIU}, 4354$, respectively. In 2019, Yang et al. [23] proposed a structure consisting of two symmetrical triangular waveguides and a ring split ring resonator cavity (CSRRC). Its design is under a maximum sensitivity of $1500 \mathrm{~nm} / \mathrm{RIU}$, the highest FOM is 65.2, but the FOM is not high enough. The above studies have controlled the change of Fano resonance peak by changing the geometric parameters of the structure and the refractive index of the medium. However, most current systems only generate a single Fano resonance and cannot simultaneously obtain high sensitivity and high FOM multi-tuned Fano resonance. Besides, the researchers used experiments to prove that various structures of plasmonic or nanophotonic sensors have high sensitivity. For example, plasma nanorod metamaterials [24], plasma gold mushroom arrays [25], single-phase nanostructures [26], hyperbolic metamaterials [27], nanoporous gold materials [28] and other sensors are proposed. These sensors have high sensitivity, but they need complex preparation methods and expensive optical equipment, so they are difficult to be miniaturized and integrated. At the same time, the above FOM is also low.

In this paper, we design a MIM waveguide structure composed of inverted U-shaped and triangular groove cavities. When TM wave is incident along the MIM waveguide, the SPP generated on the metal surface is coupled to the inverted U-shaped resonator through the F-P cavity, thus causing sharp asymmetry Fano resonance. The numerical simulation is then performed using the finite element method (FEM) to research the impact of geometric parameters and dielectric refractive index on the transmission spectrum, which realizes the tuning of multiple Fano resonance peaks. By optimizing this structure's parameters, the results show that high sensitivity and a figure of merit can be achieved, so the structure can be used to design multi-channel nanosensor devices.

\section{Model and Theoretical Analysis}

The plasmonic sensor consists of an inverted U-shaped resonant cavity and a triangular groove cavity; the schematic structural diagram is shown in Figure 1a. The yellow part represents metallic silver $\left(\varepsilon_{m}\right)$; the white part represents the air $\left(\varepsilon_{d}=1\right)$. As for geometrical parameters include the height of the inverted U-shaped resonant cavity $\mathrm{H}=240 \mathrm{~nm}$, the width of the triangular groove cavity $w_{t}=100 \mathrm{~m}$, its height $\mathrm{h}=100 \mathrm{~nm}$, the length of the groove cavity $\mathrm{L}=500 \mathrm{~nm}$, the coupling distance between the input/output waveguide and the triangular groove cavity $g_{1}=10 \mathrm{~nm}$, the coupling distance between the inverted U-shaped resonant cavity and the triangular groove cavity $g_{2}=12 \mathrm{~nm}$. To ensure that only the TM mode is propagated in the MIM waveguide, the width of the straight waveguide $\mathrm{w}$ is fixed to $50 \mathrm{~nm}$. The FEM calculates the optical response feature and steady magnetic field distribution of the structure. The scattering boundary is using as the boundary condition. The light source incident at the left port and output at the right port, solve the partial differential equation and carry out simulation calculation to obtain the transmission spectrum. The proposed structure is simple. Firstly, the metal silver layer was deposited on the silicon substrate by chemical vapor deposition. Then, the MIM waveguide and resonant cavity were etched by electron beam etching. The measured medium was injected into the waveguide resonant cavity by capillary attraction and finally sealed with high-temperature 
resistant sealant. The relative dielectric constant of the silver can be expressed by the Drude model [19]:

$$
\varepsilon_{m}(\omega)=\varepsilon_{\infty}-\frac{\omega_{p}^{2}}{\omega^{2}+i \omega \gamma}
$$

Here, $\varepsilon_{\infty}=3.7, \omega_{p}=9.1 \mathrm{eV}, \gamma=0.018 \mathrm{eV}, \varepsilon_{\infty}$ is an infinite dielectric constant, $\omega_{P}$ is the plasmonic oscillation frequency, $\omega$ is the frequency of electromagnetic radiation (incident light), $i$ is an imaginary unit, $\gamma$ is the characteristic collision frequency. When there is no triangular cavity, the transmission spectrum forms form a narrow resonance peak, as shown by the black dotted line in Figure 1b. When there is no inverted U-shaped resonator, the transmission spectrum forms a broad resonance peak, as shown by the red dotted line in Figure 1b. When the waveguide with F-P cavity is coupled with an inverted U-shaped cavity, a narrow asymmetric Fano resonance will be produced, as shown by the blue line in Figure 1b. The standing wave theory can be used to determine the resonance conditions of the F-P cavity and the inverted U-shaped resonator, as shown in the following formula [29]:

$$
\lambda=\frac{2 \operatorname{Re}\left(n_{e f f}\right) L p}{n-\varphi / 2 \pi} n=1,2,3 \ldots
$$

Here, $n$ represents the order of standing wave resonance, $L p$ is the resonator's perimeter, and $\varphi$ is the phase change caused by SPP reflection in the MIM waveguide. $\operatorname{Re}\left(n_{e f f}\right)$ is the real part of the effective refractive index $\left(n_{e f f}\right), n_{e f f}$ can be expressed as:

$$
n_{e f f}=\sqrt{\left[\varepsilon_{m}+\left(\frac{k}{k_{0}}\right)^{2}\right]}
$$

Here, $k=2 \pi / \lambda$ is the wave vector in the waveguide.

(a)

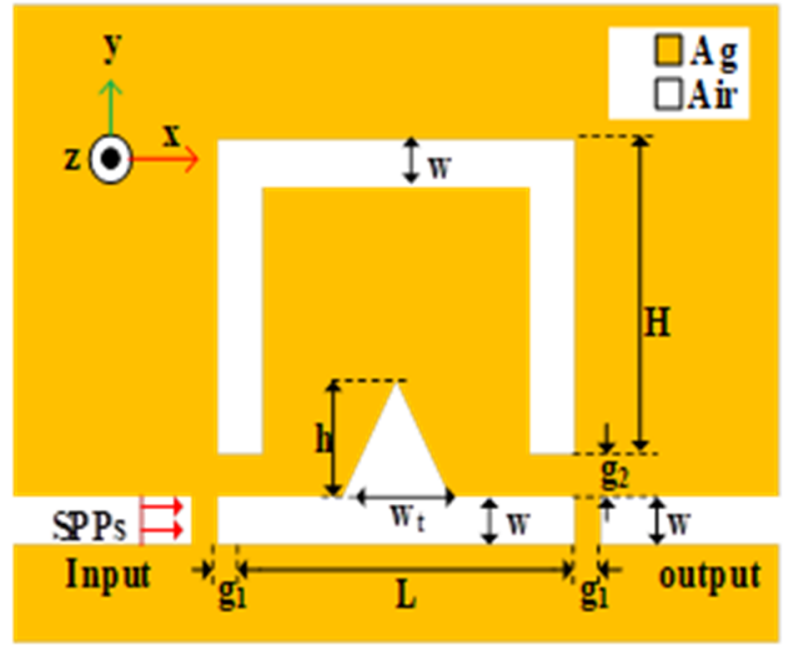

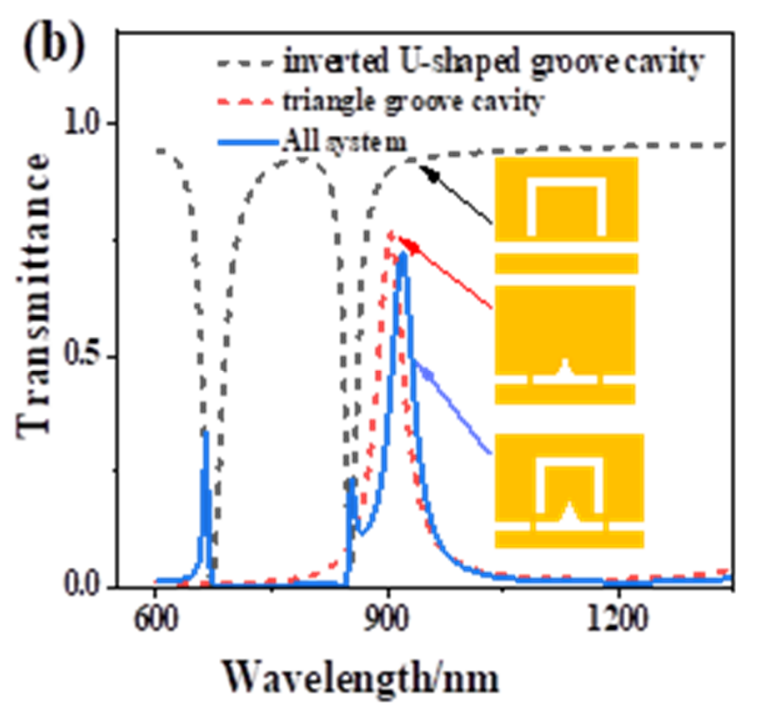

Figure 1. A plasmonic sensor and its transmission spectrum (a) Two-dimensional (2D) structure diagram; (b) Transmission spectrum of three different structures.

The Fano resonance phenomenon is analyzed based on the coupled-mode theory, the amplitudes of surface plasmons generated at the input and output ports are expressed as $S_{1+}, S_{1-}, S_{2+}, S_{2-}$ when the light wave with frequency $\omega$ is transmitted into the system only at the input port, then $S_{2+}=0 . \tau_{n_{0}}$ is the decay time of the internal loss of the FabryPerot (F-P) resonator and the inverted U-shaped resonator. $\tau_{n 1}, \tau_{n 2}$ is the attenuation time 
of the coupling between the resonant cavity and the input/output waveguide, respectively. The expression of amplitude variation with time is as follows [30]:

$$
\begin{gathered}
\frac{d A_{n}}{d t}=\left(-j \omega n-\frac{1}{\tau_{n 0}}-\frac{1}{\tau_{n 1}}-\frac{1}{\tau_{n 2}}\right) A_{n}+k_{n 1} S_{n, 1+}+k_{n 2} S_{n, 2+} \\
S_{1-}=-S_{1+}+\sum_{n} k_{n 1}^{*} A_{n}, k_{n 1}=\sqrt{\frac{2}{\tau_{n 1}}} e^{j \theta n 1} \\
S_{2-}=-S_{2+}+\sum_{n} k_{n 2}^{*} A_{n}, k_{n 2}=\sqrt{\frac{2}{\tau_{n 2}}} e^{j(\theta n 2-\phi n)} \\
S_{n, 1+}=\gamma_{n 1} e^{j \varphi n 1} S_{1+}, S_{n, 1+}=\gamma_{n 2} e^{j \varphi n 2} S_{2+} \\
\varphi_{n}=\varphi_{n 1}+\phi_{n}+\theta_{n 1}-\theta_{n 2}
\end{gathered}
$$

where $\omega_{n}$ is the resonant frequency of each resonant cavity, $k_{n i}(i=1,2)$ the coupling coefficient between each resonant cavity; $\theta_{n i}(i=1,2)$ the coupling phase between the resonator and the input-output waveguide; $\gamma_{n 1} e^{j \varphi n 1}, \gamma_{n 2} e^{j \varphi n 2}$ the standard coefficient (where $\gamma_{n 1}=\gamma_{n 2}=1$ ); $\varphi_{n}$ the total coupling phase difference of each resonant mode.

When the waveguide has the same width and are symmetrically distributed on both sides of the resonant cavity, we can get $\tau_{n}=\tau_{n 1}=\tau_{n 2}, \theta_{n 1}=\theta_{n 2}$. According to the previous calculation formula, the transmittance is obtained as follows:

$$
\mathrm{T}=\left|\frac{S_{2-}}{S_{1+}}\right|^{2}=\left|\sum_{n} \frac{2 \gamma_{n 1} e^{j \varphi n}}{-j\left(\omega-\omega_{n}\right) \tau_{n}+2+\frac{\tau_{n}}{\tau_{n o}}}\right|^{2}
$$

\section{Results and Discussion}

The reflection and transmission spectrum obtained from a plasmonic sensor is shown in Figure 2a. At the wavelengths of 664, 854, and $1250 \mathrm{~nm}$, there are three sharp asymmetric Fano resonance peaks (denoted as FR1, FR2, and FR3) in the transmission spectrum, and their transmittance is $0.33,0.23$ and 0.0136 , respectively. At the wavelengths of $920 \mathrm{~nm}$, a linear symmetrical non-sharp resonance peak (named FP) appeared in the transmission spectrum, and its transmittances were 0.72 . Next, we study the characteristics of the Fano resonance peaks of the sensor and analyze the magnetic field intensity distribution of the resonance peaks (FR1, FR2, FR3, FP), as shown in Figure 2b-e.

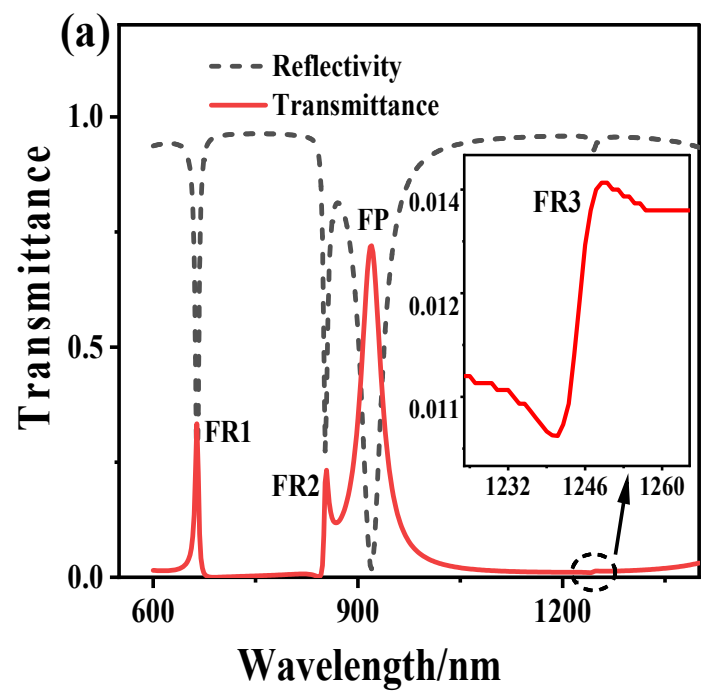

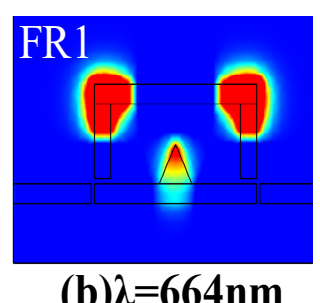
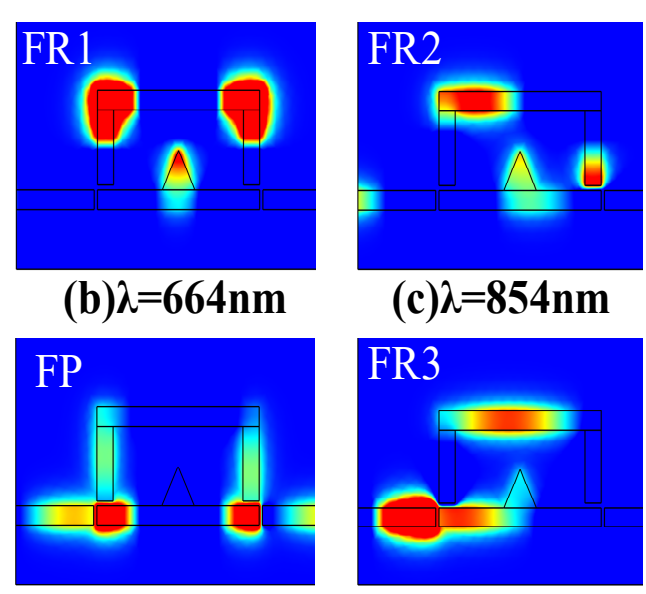

(d) $\lambda=920 \mathrm{~nm}$

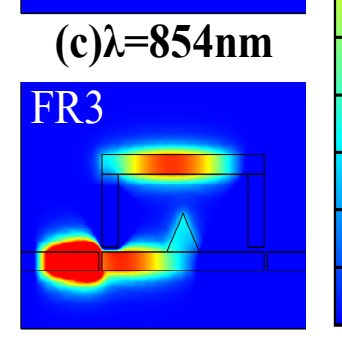

(e) $\lambda=1250 \mathrm{~nm}$

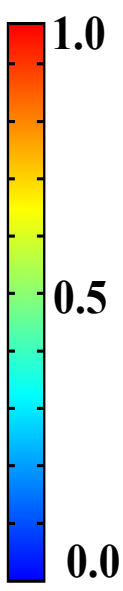

0.0

Figure 2. Transmission spectrum and magnetic field intensity distribution of plasmonic sensor (a) Reflection and transmission spectrum (b-e) the magnetic field intensity distribution of FR1, FR2, FP, and FR3. 
We can conclude from Figure 2b,c, magnetic field intensity of FR1 and FR2 resonance peaks mostly concentrate in the inverted U-shaped resonant cavity at $\lambda=664 \mathrm{~nm}$, $\lambda=854 \mathrm{~nm}$, and only a small amount of energy is distributed in the triangular groove cavity. It can be found that when $\lambda=664 \mathrm{~nm}$, the magnetic field intensity at the two cavities coupling is in the same direction. We can conclude from Figure $2 d$, the magnetic field intensity of FP1 at $\lambda=920 \mathrm{~nm}$. We can see from the figure that most of the energy are concentrated in the input-output waveguide and only a small amount of energy is coupled to the inverted U-shaped cavity. As shown in Figure 2e, the magnetic field intensity of FP1 resonance peak at $\lambda=1250 \mathrm{~nm}$. We can see from the figure that most of the magnetic field energy is distributed in the left input waveguide and a few in the inverted U-shaped cavity, for the reason of relatively low transmittance. In FR1, FR2, and FR3, the antinodes are distributed in a large proportion in the inverted U-shaped resonator, which has a strong magnetic field distribution. The energy in the resonant cavity is difficult to be transmitted to the waveguide. As a result, SPP is not output to the output waveguide, so Fano resonance occurs, and the transmittance is low. The antinode of the FP distributes considerable energy in the MIM waveguide, and it is difficult for SPP to couple into the inverted U-shaped cavity, so no Fano resonance peak is generated.

Firstly, the influence of common metals such as gold $(\mathrm{Au})$, silver $(\mathrm{Ag})$, and aluminum (Al) on the transmission spectrum of the structure is studied. As shown in Figure 3, it can be seen that the transmittance of $\mathrm{Ag}$ is higher than that of $\mathrm{Au}$ and $\mathrm{Al}$, so it can be concluded that the loss of $\mathrm{Ag}$ is the least. Based on this, we use $\mathrm{Ag}$ in this structure. We study the optical response of the Fano resonance by changing the parameters of the structure. First, we peer the influence of the height (h) of the triangular cavity on the transmission spectrum. When other parameters remain unchanged, $\mathrm{h}$ is scanned from 80 to $120 \mathrm{~nm}$ with a scanning interval of $10 \mathrm{~nm}$. The transmission spectrum is shown in Figure 4a. The FOM as functions of $h$ in Figure $4 b$. We can see from the picture that with the increase of $h$, the transmittance of FR1 and FR2 decreased and the line type of Fano resonance changed. Under the condition that other parameters remain unchanged, we study the effect of the width $\left(w_{t}\right)$ of the triangular cavity on the transmission spectrum. When $w_{t}$ is scanned from 80 to $120 \mathrm{~nm}$ with a scanning interval of $10 \mathrm{~nm}$, its transmission spectrum is shown in Figure $4 \mathrm{c}, \mathrm{FOM}$ as functions of $w_{t}$ in Figure $4 \mathrm{~d}$. We can see from the picture that with the increase of $w_{t}$ the transmittance of FR1, FR2 increases, and the line type of Fano resonance changes. The above changes are mainly because the wavelength of the Fano resonant peaks is close to the dark mode's resonant wavelength, which is primarily affected by the dark mode. In contrast, the change of the triangular cavity mainly affects the resonant wavelength of FP.

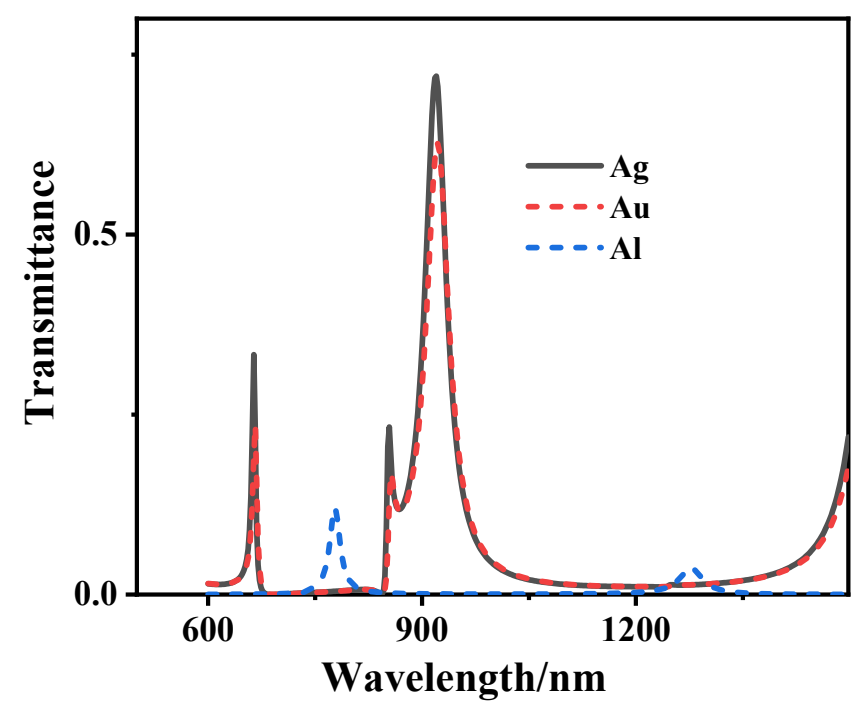

Figure 3. Transmission spectra of $\mathrm{Ag}, \mathrm{Au}, \mathrm{Al}$. 

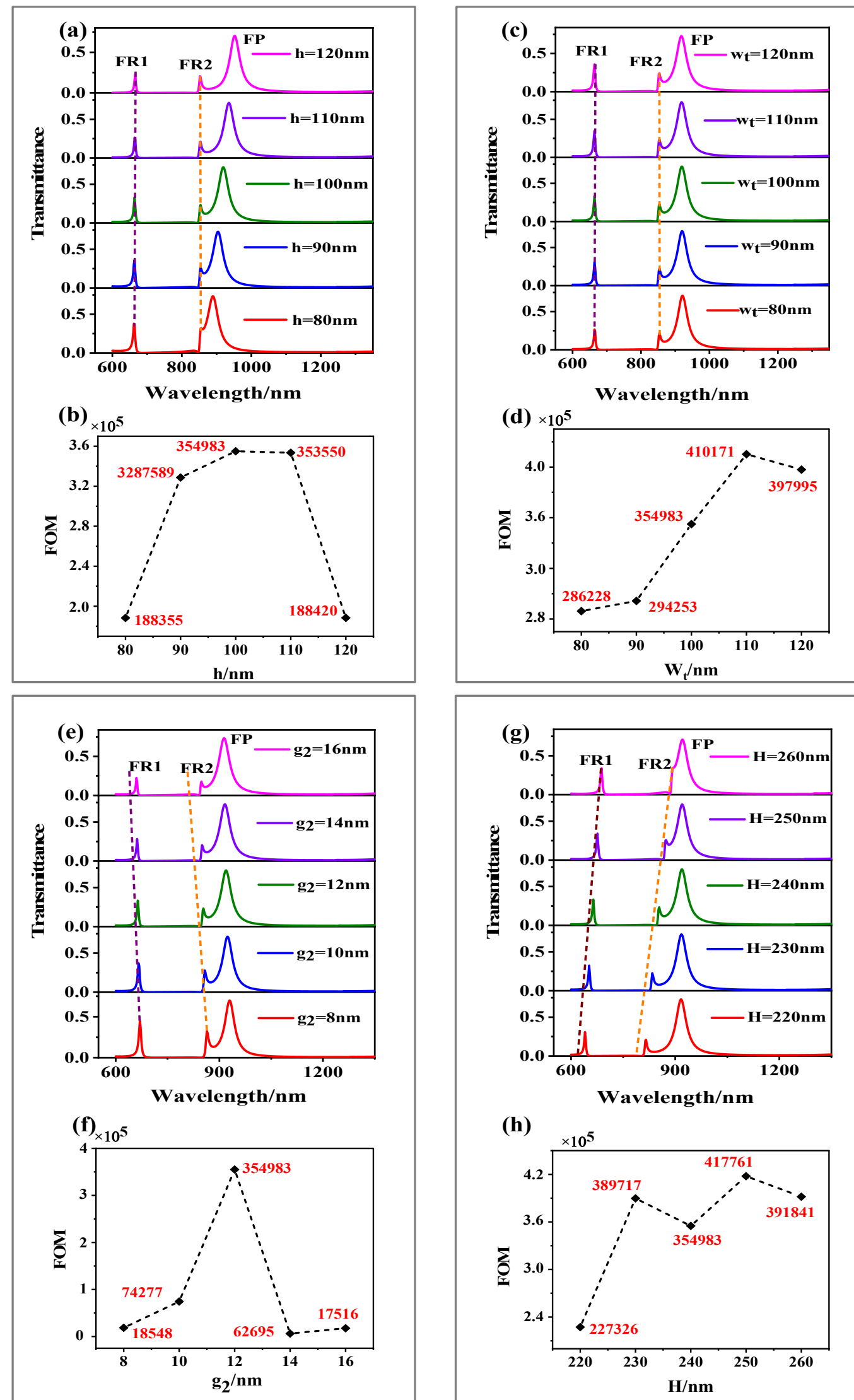

(h)

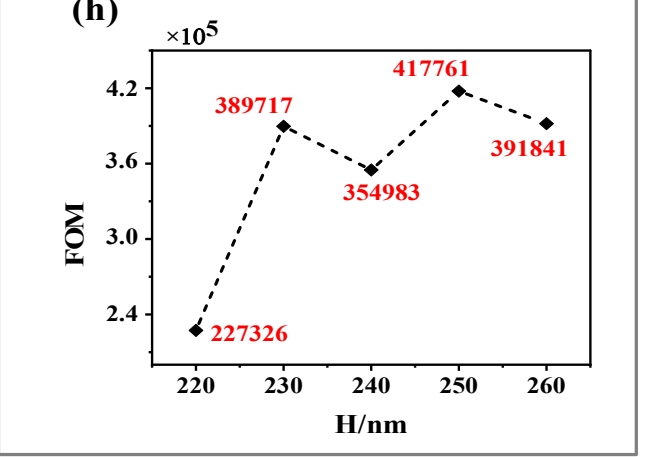

Figure 4. The effect of geometric parameters on the transmission spectrum (a) h, (c) $w_{t},(\mathbf{e}) g_{2}$ and (g) $\mathrm{H}$ as a function of the wavelength; FOM as a function of (b) h, (d) $w_{t},(\mathbf{f}) g_{2}$ and (h) $\mathrm{H}$. 
Then, the effect of the coupling distance $\left(g_{2}\right)$ between the inverted U-shaped cavity and the triangular groove cavity on the optical properties of Fano resonance is under our research. When other parameters remain unchanged, $g_{2}$ is scanned from 8 to $16 \mathrm{~nm}$ with a scanning interval of $2 \mathrm{~nm}$, its transmission spectrum is shown in Figure 4e, FOM as functions of $g_{2}$ in Figure $4 \mathrm{f}$. It can be observed that with the increase of $g_{2}$, the transmittance of FR1 and FR2 decreases, Fano resonance peak appear linear blue shift phenomenon and the line type of Fano resonance changed. Due to the increasing value of $g_{2}$, the coupling strength between the inverted $\mathrm{U}$-shaped resonator and the triangular groove cavity is weakened. Finally, under the condition that other parameters remain unchanged, the height $(\mathrm{H})$ of the inverted U-shaped resonator is increased from 220 to $260 \mathrm{~nm}$, performing a parameter scan with an interval of $10 \mathrm{~nm}$. The transmission spectrum is shown in Figure $4 \mathrm{~g}$. The FOM as functions of $\mathrm{H}$ in Figure $4 \mathrm{~h}$. We can see that the transmittance of FR1 and FR2 increases and shows an apparent linear redshift, and the Fano resonance peak line shape also changes. This is because the height of the inverted U-shaped resonator is increasing, which leads to an increasing in Lp. According to Formula (2), it can be obtained that the resonance wavelength also moves in the long-wavelength direction.

Finally, we discuss the sensor's characteristics, in which sensitivity $(S)$ is a crucial parameter to evaluate the sensor's attributes. It can be expressed as: $S=d \lambda / d n(\mathrm{~nm} / R I U)$ [22], where $d n$ is the change of refractive index, $d \lambda$ is the change of the resonance wavelength. Changing the white part of the structure in Figure $1 \mathrm{a}$ is changing the $\mathrm{n}$ of the medium. When the refractive index increases from 1.00 to 1.10 and the scanning interval of 0.02 , the transmission spectrum is shown in Figure 5a. It can be seen from the figure that there is a noticeable linear redshift in the resonance wavelength with the increase of $n$. Figure $5 b$ describes the relationship between the $n$ and the resonance wavelength. The maximum $S$ of the structure is $840 \mathrm{~nm} / \mathrm{RIU}$.
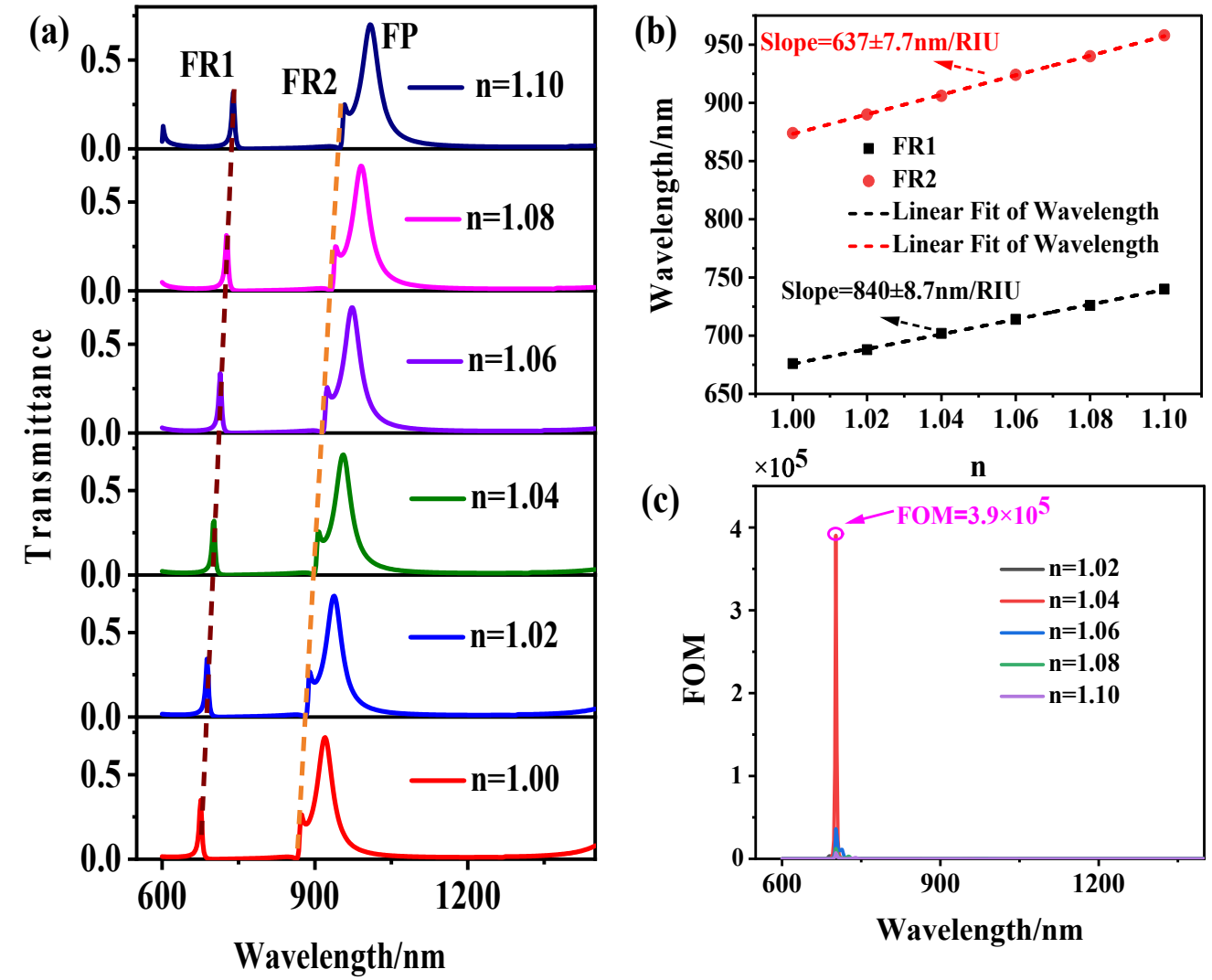

Figure 5. The effect of different $\mathrm{n}$ on the sensing performance (a) The influence of varying $n$ on the transmission spectrum (b) Fitting curve of resonance wavelength and refractive index $n$ (c) The FOM distribution with the wavelength of the structure. 
Moreover, the figure of merit (FOM) is another critical feature for evaluating sensor characteristics. It can be expressed as: $F O M=\Delta \mathrm{T} / \mathrm{T} \Delta n$ [31], where $\mathrm{T}$ is the transmittance of the system, $\Delta \mathrm{T} / \Delta n$ indicates the change of transmittance caused by the transformation of the refractive index of the system in the wavelength range. The FOM distribution of the wavelength of the structure is shown in Figure 5c. Due to the sharpness of the Fano resonance peaks, according to the formula in the figure of merit, it is calculated that when $\lambda=702 \mathrm{~nm}$, the structure can obtain the maximum figure of merit (FOM) of $3.9 \times 10^{5}$. Therefore, compared with previous technologies, our design has greater sensitivity and the highest FOM, as shown in Table 1.

Table 1. A comparison of the sensing characteristics of this work with previous work.

\begin{tabular}{ccccc}
\hline Reference & Year & $\begin{array}{c}\text { S } \\
\text { (nm/RIU) }\end{array}$ & FOM & Structure \\
\hline$[21]$ & 2016 & 596 & 7.5 & Double rectangular cavity \\
{$[22]$} & 2017 & 718 & 4354 & Asymmetric ring cavity \\
{$[31]$} & 2014 & 600 & 650 & Rectangular cavity \\
{$[32]$} & 2016 & 600 & 3803 & Two rectangular cavity \\
{$[33]$} & 2017 & 750 & 68.3 & Trapezoid cavity \\
{$[34]$} & 2018 & 497.8 & 480 & Plasmonic metasurface \\
{$[35]$} & 2018 & 680 & 8.68 & T shaped cavity \\
{$[36]$} & 2018 & 350 & 15 & Hybrid Metasurface \\
{$[37]$} & 2019 & 780 & $1.56 \times 10^{5}$ & M-type resonant cavity \\
{$[38]$} & 2020 & 540 & 101.3 & Elliptical cavity \\
This work & 2020 & 840 & $3.9 \times 10^{5}$ & Inverted U cavity \\
\hline
\end{tabular}

Then, the medium of the structure is filled with ethanol material to realize the temperature sensor. The refractive index of ethanol can be defined as [39]:

$$
n=n_{0}-\mathrm{d} n / d \mathrm{~T}\left(\mathrm{~T}-\mathrm{T}_{0}\right)
$$

where, $\mathrm{T}_{0}$ is the ambient temperature at $20^{\circ} \mathrm{C}$, $\mathrm{T}$ is the ambient temperature, $n_{0}=1.36084$, $\mathrm{d} n / d \mathrm{~T}=3.94 \times 10^{-4}$. Figure 6a shows the transmission spectra at different ambient temperatures. It can be seen from the figure that the resonance wavelength shows a blueshift with the increase of T. According to the formula of ethanol refractive index, with the rise of $T$, the refractive index $n$ of ethanol significantly decreases. Figure $6 \mathrm{~b}$ is the linear curve of the relationship between the ambient temperature $\mathrm{T}$ and the resonance wavelength. It can be seen that the temperature sensitivities of the three Fano resonances are $0.2 \mathrm{~nm} /{ }^{\circ} \mathrm{C}$, $0.25 \mathrm{~nm} /{ }^{\circ} \mathrm{C}, 0.33 \mathrm{~nm} /{ }^{\circ} \mathrm{C}$, respectively.

Finally, to verify that the structure is used for the biosensor, we studied water $(n=1.33)$, acetone $(n=1.358)$, 2-propanol $(n=1.377)$, and chloroform $(n=1.446)$ as the background materials. Figure 7 a shows the transmission spectra of the refractive index of different liquids, and Figure $7 \mathrm{~b}$ shows the linear curves of various refractive index and resonance wavelengths. It can be seen from the figure that the sensitivities of the three Fano formants are $516 \mathrm{~nm} / \mathrm{RIU}, 637 \mathrm{~nm} / \mathrm{RIU}$, and $828 \mathrm{~nm} / \mathrm{RIU}$, respectively. Through the verification of different liquid refractive index, it is shown that this structure has a potential application in high-sensitivity nanosensors. 

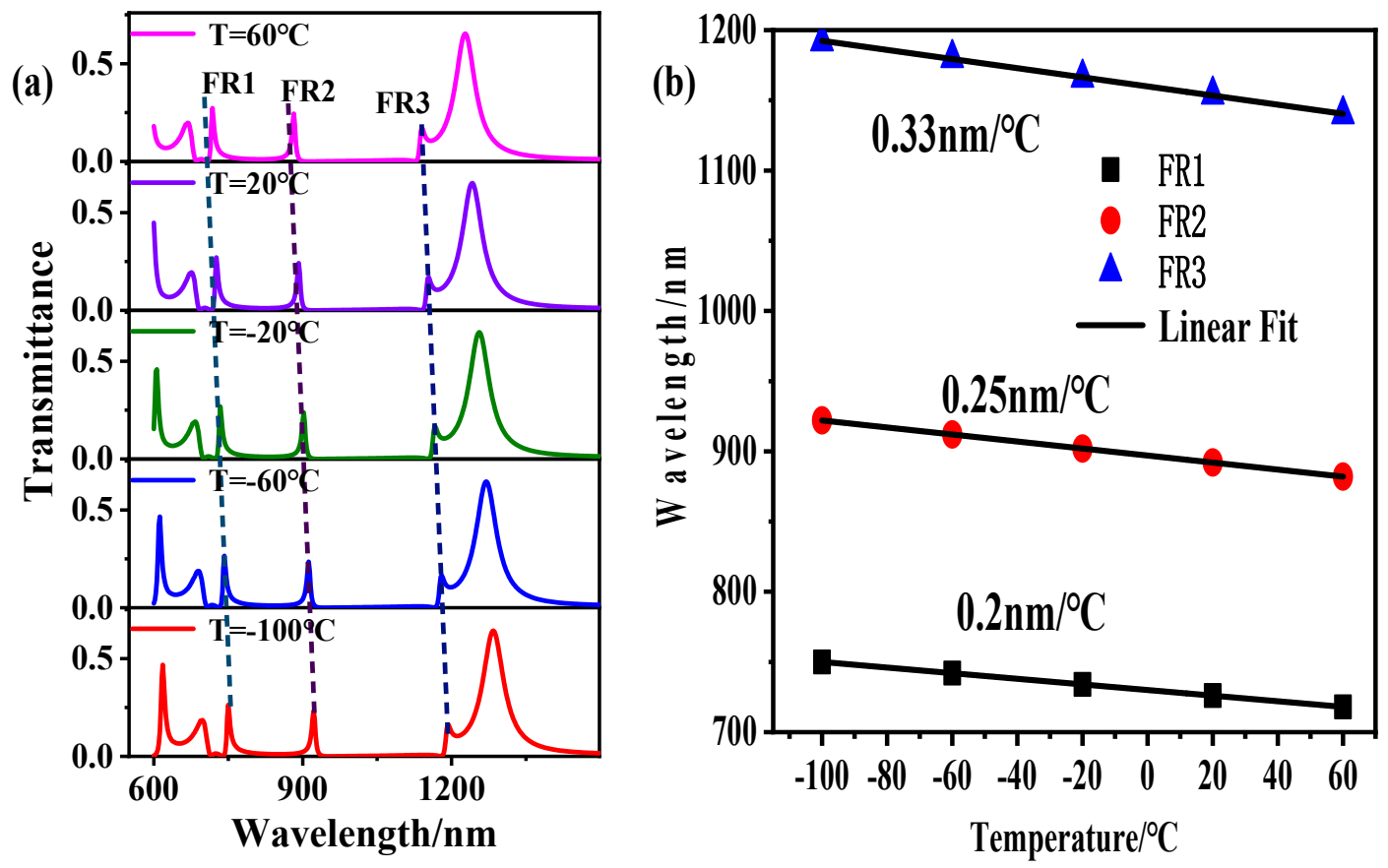

Figure 6. (a) Transmission spectra at different ambient temperatures T. (b) Linear curve of ambient temperature T and resonance wavelength.
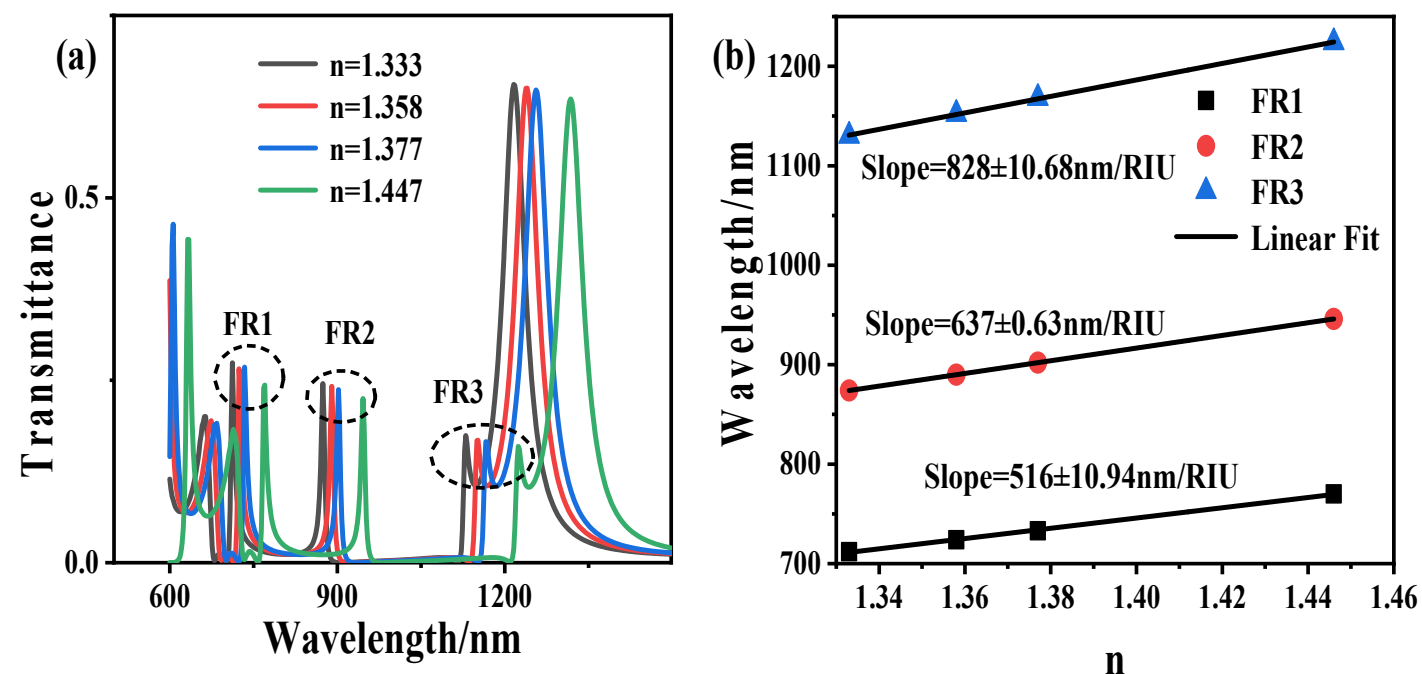

Figure 7. (a)The transmission spectra of refractive indices of different liquids. (b) The linear curves of the different refractive index and resonance wavelengths.

\section{Conclusions}

This report designs a MIM waveguide structure, which has a triangular slot cavity between the input and output waveguides, which is coupled with the inverted U-shaped resonator. The FEM is utilized to simulate Fano's optical characteristics and research the impact of the geometric structure change. The phenomenon of multiple Fano resonance is explained by multimode interference coupled-mode theory. By changing the inverted U-shaped cavity's height, the coupling distance between the inverted U-shaped cavity and triangular groove cavity, and the height and width of the triangular cavity. It can achieve the change of transmittance and the shift and line type change of Fano resonance peaks, and it has better sensing characteristics. The maximum sensitivity is $840 \mathrm{~nm} / \mathrm{RIU}$, and the highest 
figure of merit is through simulation calculation. It shows that the structure has a broad application prospect in designing plasmonic nanosensors. The sensing properties can also be realized in another style, assuming the nanostructure used to study the polarization state. By investigating these parameters, such as polarization rotation, ellipticity, and so on, we can understand polarization-based nanostructures to achieve high sensitivity. Therefore, we can consider using the polarization state to realize plasmonics nanosensors.

Author Contributions: G.X.; H.Y. and L.Z. conceived the design; Y.X. performed the simulation; Y.X. analyzed the simulation data; H.Y. wrote the paper; Z.O.; J.C.; H.L.; X.L.; L.Z., and J.L. made revisions and finalized the document. All authors have read and agreed to the published version of the manuscript.

Funding: This work is partially supported by the National Natural Science Foundation of China (61765004, 61465004,61874036), the Guangxi Natural Science Foundation (2017GXNSFAA198164, 2016GXNSFAA380006), the Guangxi Key Laboratory of Precision Navigation Technology and Application (DH202006, DH202004), the Guangxi Key Laboratory of Automatic Detecting Technology and Instruments (YQ20115), the Open Fund of Foshan University, Research Fund of Guangdong Provincial Key Laboratory of Semiconductor Micro Display (No. 2020B121202003), and Research Fund of Guangdong-Hong Kong-Macao Joint Laboratory for Intelligent Micro-Nano Optoelectronic Technology (Project No. 2020B1212030010).

Institutional Review Board Statement: Not applicable.

Informed Consent Statement: Not applicable.

Data Availability Statement: Data sharing not applicable.

Conflicts of Interest: The authors declare no conflict of interest.

\section{References}

1. Homola, J.; Yee, S.S.; Gauglitz, G. Surface plasmon resonance sensors. Sens. Actuators B Chem. 1999, 54, 3-15. [CrossRef]

2. Anker, J.N.; Hall, W.P.; Lyandres, O.; Shah, N.C.; Zhao, J.; Van Duyne, R.P. Biosensing with plasmonic nanosensors. Nanosci. Technol. 2010, 308-319. [CrossRef]

3. Xu, Y.; Bai, P.; Zhou, X.; Akimov, Y.; Png, C.E.; Ang, L.; Knoll, W.; Wu, L. Optical Refractive Index Sensors with Plasmonic and Photonic Structures: Promising and Inconvenient Truth. Adv. Opt. Mater. 2019, 7, 7. [CrossRef]

4. Sekkat, Z.; Hayashi, S.; Nesterenko, D.V.; Rahmouni, A.; Refki, S.; Ishitobi, H.; Inouye, Y.; Kawata, S. Plasmonic coupled modes in metal-dielectric multilayer structures: Fano resonance and giant field enhancement. Opt. Express 2016, 24, 20080-20088. [CrossRef] [PubMed]

5. Wang, J.; Liu, X.; Li, L.; He, J.; Fan, C.; Tian, Y.; Ding, P.; Chen, D.; Xue, Q.; Liang, E. Huge electric field enhancement and highly sensitive sensing based on the Fano resonance effect in an asymmetric nanorod pair. J. Opt. 2013, 15, 105003. [CrossRef]

6. Hayashi, S.; Nesterenko, D.V.; Sekkat, Z. Waveguide-coupled surface plasmon resonance sensor structures: Fano lineshape engineering for ultrahigh-resolution sensing. J. Phys. D Appl. Phys. 2015, 48, 325303. [CrossRef]

7. Limonov, M.F.; Rybin, M.V.; Poddubny, A.N.; Kivshar, Y.S. Fano resonances in photonics. Nat. Photonics 2017, 11, 543-554. [CrossRef]

8. Luk'Yanchuk, B.; Zheludev, N.I.; Maier, S.A.; Halas, N.J.; Nordlander, P.; Giessen, H.; Chong, C.T. The Fano resonance in plasmonic nanostructures and metamaterials. Nat. Mater. 2010, 9, 707-715. [CrossRef] [PubMed]

9. Zheng, C.; Jia, T.; Zhao, H.; Xia, Y.; Zhang, S.; Feng, D.; Sun, Z.-R. Theoretical study on narrow Fano resonance of nanocrescent for the label-free detection of single molecules and single nanoparticles. RSC Adv. 2018, 8, 3381-3391. [CrossRef]

10. Shuai, Y.; Zhao, D.; Chadha, A.S.; Seo, J.-H.; Yang, H.; Fan, S.; Ma, Z.; Zhou, W. Coupled double-layer Fano resonance photonic crystal filters with lattice-displacement. Appl. Phys. Lett. 2013, 103, 241106. [CrossRef]

11. Mao, J.; Zhai, X.; Wang, L.; Li, H. Numerical analysis of near-infrared plasmonic filter with high Figof merit based on Fano resonance. Appl. Phys. Express 2017, 10, 082201. [CrossRef]

12. Yan, S.; Zhang, M.; Zhao, X.; Zhang, Y.; Wang, J.; Jin, W. Refractive Index Sensor Based on a Metal-Insulator-Metal Waveguide Coupled with a Symmetric Structure. Sensors 2017, 17, 2879. [CrossRef]

13. Yu, S.; Zhao, T.; Yu, J.; Pan, D. Tuning Multiple Fano Resonances for On-Chip Sensors in a Plasmonic System. Sensors 2019, 19, 1559. [CrossRef] [PubMed]

14. Huang, B.; Meng, H.; Wang, Q.; Wang, H.; Zhang, X.; Yu, W.; Tan, C.; Huang, X.; Wang, F. Plasmonic-Induced Transparency and Slow-Light Effect Based on Stub Waveguide with Nanodisk Resonator. Plasmon 2016, 11, 543-550. [CrossRef]

15. Li, D.; Du, K.; Liang, S.; Zhang, W.; Mei, T. Wide band dispersionless slow light in hetero-MIM plasmonic waveguide. Opt. Express 2016, 24, 22432. [CrossRef] [PubMed] 
16. Nozaki, K.; Shinya, A.; Matsuo, S.; Sato, T.; Kuramochi, E.; Notomi, M. Ultralow-energy and high-contrast all-optical switch involving Fano resonance based on coupled photonic crystal nanocavities. Opt. Express 2013, 21, 11877-11888. [CrossRef] [PubMed]

17. Zayats, A.V.; Smolyaninov, I.I.; Maradudin, A.A. Nano-optics of surface plasmon polaritons. Phys. Rep. 2005, 408, 131-314. [CrossRef]

18. Zhang, Y.X.; Zhang, Y.; Mølmer, K. Surface Plasmon Launching by Polariton Superradiance. ACS Photonics 2019, 6, 871-877. [CrossRef]

19. Lin, X.-S.; Huang, X. Tooth-shaped plasmonic waveguide filters with nanometeric sizes. Opt. Lett. 2008, 33, 2874-2876. [CrossRef]

20. Gramotnev, D.K.; Bozhevolnyi, S.I. Plasmonics beyond the diffraction limit. Nat. Photonics 2010, 4, 83-91. [CrossRef]

21. Zhang, Z.; Luo, L.; Xue, C.; Zhang, W.; Yan, S. Fano Resonance Based on Metal-Insulator-Metal Waveguide-Coupled Double Rectangular Cavities for Plasmonic Nanosensors. Sensors 2016, 16, 642. [CrossRef] [PubMed]

22. Zhao, X.; Zhang, Z.; Yan, S. Tunable Fano Resonance in Asymmetric MIM Waveguide Structure. Sensors 2017, 17, 1494. [CrossRef] [PubMed]

23. Yang, X.; Hua, E.; Wang, M.; Wang, Y.; Wen, F.; Yan, S. Fano Resonance in a MIM Waveguide with Two Triangle Stubs Coupled with a Split-Ring Nanocavity for Sensing Application. Sensors 2019, 19, 4972. [CrossRef]

24. Kabashin, A.V.; Evans, P.; Pastkovsky, S.; Hendren, W.; Wurtz, G.A.; Atkinson, R.; Pollard, R.; Podolskiy, V.A.; Zayats, A.V. Plasmonc nanorod metamaterials for biosensing. Nat. Mater. 2009, 8, 867-871. [CrossRef] [PubMed]

25. Shen, Y.; Zhou, J.; Liu, T.; Tao, Y.; Jiang, R.; Liu, M.; Xiao, G.; Zhu, J.; Zhou, Z.-K.; Wang, X.; et al. Plasmonic gold mushroom arrays with refractive index sensing figures of merit approaching the theoretical limit. Nat. Commun. 2013, 4, 2381. [CrossRef] [PubMed]

26. Kravets, V.G.; Schedin, F.; Jalil, R.; Britnell, L.; Gorbachev, R.V.; Ansell, D.; Thackray, B.; Novoselov, K.S.; Geim, A.K.; Kabashin, A.V.; et al. Singular phase nano-optics in plasmonic metamaterials for label-free single-molecule detection. Nat. Mater. 2013, 12, 304-309. [CrossRef] [PubMed]

27. Sreekanth, K.V.; Alapan, Y.; ElKabbash, M.; Ilker, E.; Hinczewski, M.; Gurkan, U.A.; De Luca, A.; Strangi, G. Extreme sensitivity biosensing platform based on hyperbolic metamaterials. Nat. Mater. 2016, 15, 621-627. [CrossRef]

28. Garoli, D.; Calandrini, E.; Giovannini, G.; Hubarevich, A.; Caligiuri, V.; De Angelis, F. Nanoporous gold metamaterials for high sensitivity plasmonic sensing. Nanoscale Horiz. 2019, 4, 1153-1157. [CrossRef]

29. Li, Z.; Wen, K.; Chen, L.; Lei, L.; Zhou, J.; Zhou, D.; Fang, Y.; Wu, B. Refractive index sensor based on multiple Fano resonances in a plasmonic MIM structure. Appl. Opt. 2019, 58, 4878-4883. [CrossRef]

30. Li, S.; Wang, Y.; Jiao, R.; Wang, L.; Duan, G.; Yu, L. Fano resonances based on multimode and degenerate mode interference in plasmonic resonator system. Opt. Express 2017, 25, 3525-3533. [CrossRef]

31. Qi, J.; Chen, Z.; Chen, J.; Li, Y.; Qiang, W.; Xu, J.; Qian, S. Independently tunable double Fano resonances in asymmetric MIM waveguide structure. Opt. Express 2014, 22, 14688-14695. [CrossRef] [PubMed]

32. Li, S.; Zhang, Y.; Song, X.; Wang, Y.; Yu, L. Tunable triple Fano resonances based on multimode interference in coupled plasmonic resonator system. Opt. Express 2016, 24, 15351-15361. [CrossRef] [PubMed]

33. Zhou, J.; Chen, H.; Zhang, Z.; Tang, J.; Cui, J.; Xue, C.; Yan, S. Transmission and refractive index sensing based on Fano resonance in MIM waveguide-coupled trapezoid cavity. Aip Adv. 2017, 7, 015020. [CrossRef]

34. Alipour, A.; Farmani, A.; Mir, A. High Sensitivity and Tunable Nanoscale Sensor Based on Plasmon-Induced Transparency in Plasmonic Metasurface. IEEE Sens. J. 2018, 18, 7047-7054. [CrossRef]

35. Wang, L.; Zeng, Y.P.; Wang, Z.Y.; Xia, X.P.; Liang, Q.Q. A refractive index sensor based on an analogy T shaped metal-insulatormetal waveguide. Optik 2018, 172, 1199-1204. [CrossRef]

36. Rifat, A.A.; Rahmani, M.; Xu, L.; Miroshnichenko, A.E. Hybrid metasurface based tunable near-perfect absorber and plasmonic sensor. Materials 2018, 11, 1091. [CrossRef]

37. Qiao, L.; Zhang, G.; Wang, Z.; Fan, G.; Yan, Y. Study on the Fano resonance of coupling M-type cavity based on surface plasmon polaritons. Opt. Commun. 2019, 433, 144-149. [CrossRef]

38. El Haffar, R.; Farkhsi, A.; Mahboub, O. Optical properties of MIM plasmonic waveguide with an elliptical cavity resonator. Appl. Phys. A 2020, 126, 1-10. [CrossRef]

39. Chen, F.; Zhang, H.; Sun, L.; Li, J.; Yu, C. Temperature tunable Fano resonance based on ring resonator side coupled with a MIM waveguide. Opt. Laser Technol. 2019, 116, 293-299. [CrossRef] 